\title{
A regionalização da atenção psicossocial em álcool e outras drogas no Brasil
}

\section{Regionalization of psychosocial care of alcohol and other drug addiction in Brazil}

\section{La regionalización de atencón psicosocial en alcohol y otras drogas en Brasil}

\author{
João Paulo Macedo ${ }^{1}$ \\ Mariana Marinho de $\mathrm{Abreu}^{2}$ \\ Magda Dimenstein ${ }^{3}$
}

\begin{abstract}
RESUMO: Esse trabalho objetiva discutir o processo de regionalização da RAPS no âmbito da atenção às pessoas com necessidades decorrentes ao uso de álcool, crack e outras drogas. Especificamente, pretende dimensionar os pontos de atenção da RAPS voltados para essa problemática, além de apontar os desafios quanto ao processo de expansão e de interiorização da atenção psicossocial no Brasil. É um estudo transversal, delineado a partir da elaboração de um banco de dados com base nas informações disponibilizadas pela Coordenação Nacional de Saúde Mental e pela plataforma "Observatório Crack é possível vencer". As informações foram reconstruídas a partir do banco de indicadores da plataforma Regiões e Redes de modo a relacionar os 5.570 municípios, organizados em 438 regiões de saúde. As unidades de análise foram, no caso o mapeamento dos serviços da RAPS, a estratificação por estado e porte dos municípios, e no que diz respeito ao processo de Regionalização da Atenção ao álcool e outras drogas no Brasil, as próprias regiões de saúde (CIR). Esse dimensionamento incluiu a rede intersetorial (CRAS, CREAS, Centro Pop e CRR). Observou-se uma rede de serviços insuficiente e pouco organizada. Há uma "expansão errante" que não considera o planejamento e a pactuação regional necessários para a sustentabilidade do sistema. Há vazios assistenciais em todo o país em relação à atenção ao usuário de álcool, crack e outras drogas, fato que vem provocando o incremento desenfreado das comunidades terapêuticas. Há uma desigualdade evidente na distribuição dos serviços nos municípios de grande e de pequeno portes.
\end{abstract}

Palavras-chave: regionalização, RAPS, atenção psicossocial, álcool e outras drogas.

\begin{abstract}
This work aims to discuss the RAPS regionalization process in regard to the care of people with needs from alcohol, crack and other drug use. Specifically, it intends to give dimension to the care points of the RAPS dedicated to this issue, and point out the challenges of the expansion and interiorization processes in psychosocial care in Brazil. It's a cross-study, defined from the elaboration of a database which uses the info made available by the Coordenação Nacional de Saúde Mental (National Coordination of Mental Health) and the "Observatório Crack é possível vencer"

\footnotetext{
1 Professor dos Programas de Pós-Graduação em Psicologia e em Políticas Públicas da Universidade Federal do Piauí, Natal, Rio Grande do Norte, Brasil. E-mail: jpmacedo@ufpi.edu.br

2 Bolsista de Iniciação Científica. Aluna do Curso de Psicologia na Universidade Federal do Piauí (UFPI). Parnaíba, Piauí, Brasil. E-mail: mariimarinhoa@gmail.com

3 Professora Titular do Programa de Pós-Graduação em Psicologia da Universidade Federal do Rio Grande do Norte (UFRN). Natal, Rio Grande do Norte, Brasil. E-mail: magda@ufrnet.br
} Parnaíba-PI. Mestre e Doutor em Psicologia pelo PPgPsi da Universidade Federal do Rio Grande do Norte (UFRN).
\end{abstract}

ISSN 1982-8829 Tempus, actas de saúde colet, Brasília, 11(3), 144-162, 2017 - Epub mar, 2018 
("Crack Watch: A win is possible") platform. The pieces of information were reconstructed from the indicators database of the Regions and Networks platform as to relate the 5.570 municipalities, organized in 438 health regions. The unity analysis was, in the case of mapping RAPS services, the state by state stratification and municipalities size, as well as to what concerns the regionalization of alcohol and other drugs care policy in Brazil, the very regions of health (CIR). This dimension included the intersectional network (CRAS, CREAS, Centro Pop e CRR). An insufficient and not very organized network of services was found. There is a wandering expansion that does not consider the necessary planning and the regional pact for the sustainability of the system. There are assistential gaps all over the country in relation to care of alcohol, crack and other drugs, a fact that has caused the uncontrolled growth of therapeutic communities. There is an evident inequality in the distribution of services in municipalities of large and smaller size.

Keywords: regionalization, RAPS, psychosocial attention, alcohol and drugs.

RESUMEN: Este trabajo pretende discutir el proceso de regionalización de la RAPS - Red de atención psicosocial - en el ámbito de atención a las personas con necesidades decurrentes al uso del alcohol, craque y otras drogas. Específicamente, pretende dimensionar los puntos de atención de la RAPS volcados para esta problemática, además de señalar los desafíos en lo que concierne al proceso de la expansión y de la interiorización de la atención psicosocial en Brasil. Este es un estudio transversal, delineado a partir de la elaboración de un banco de datos con base en las informaciones disponibles por la Coordinación Nacional de Salud Mental y por la plataforma "Observatório Crack é possivel vencer". Las informaciones fueron reconstruidas a partir del banco de indicadores de la plataforma "Regiões e Redes" en la que se articulan los 5.570 municipios brasileños, organizados en 438 regionales de salud. Las unidades de análisis fueron, específicamente, el mapeamiento de los servicios de la RAPS, la estratificación por estado y el tamaño de los municipios, y en lo que se refiere, particularmente, al proceso de la Regionalización de la Atención en alcohol y otras drogas en Brasil, las propias regionales de salud (CIR - Comisiones Integrales Regionales). Este dimensionamiento incluye la red intersectorial (CRAS - Centros de referencias de la Asistencia Social, CREAS - Centros de Referencia Especializados de Asistencia Social, Centro Pop Centro de Referencia para Población en Situación Ambulante, y el CRR - Centro de Referencia Especializado en Craque y Alcohol). Se observó una red de servicios insuficiente y poco organizada. Hay una "expansión errante" que no considera la planeación y el pacto regional necesario para la sostenibilidad del sistema. Hay vacíos asistenciales en todo el país en relación con la atención del usuario de alcohol, craque y otras drogas, hecho que ha provocado el incremento desenfrenado de las comunidades terapéuticas. Hay una desigualdad evidente en la distribución de los servicios en los municipios, sean estos grandes o pequeños.

Palabras-clave: regionalización, RAPS, atención psicosocial, alcohol y otras drogas.

\section{INTRODUÇÃO}

A temática do álcool e outras drogas nos dias atuais é considerada um problema de saúde pública em nível mundial visto que o uso abusivo e a dependência de substâncias psicoativas provocam prejuízos graves não só para as pessoas que as consomem, mas também para aquelas que estão no entorno (familiares, vizinhos, amigos, etc.). Apesar dos muitos avanços, seja no modo de compreensão da problemática, seja nas estratégias de cuidado, principalmente com a emergência do paradigma da Redução de Danos, infelizmente, as políticas públicas no Brasil estão pouco integradas e há inúmeras barreiras de acesso que acentuam as iniquidades entre pessoas com necessidades de cuidados decorrentes do álcool e outras drogas e que se encontram em extrema vulnerabilidade social ${ }^{1}$.

ISSN 1982-8829 Tempus, actas de saúde colet, Brasília, 11(3), 144-162, 2017 - Epub mar, 2018 
Após a Lei da Reforma Psiquiátrica Brasileira (Lei 10.216/01), o Ministério da Saúde estabeleceu a Política Nacional de Atenção Integral ao Uso de Álcool e Outras Drogas (PNS-AD) que representa um avanço na discussão. A PNS-AD trata dos paradigmas, estratégias e modelos de atenção respaldados na proposta de Redução de Danos, a qual prima pelo cuidado baseado na autonomia e liberdade, reconhecendo o sujeito em sua singularidade e como corresponsável pelo seu tratamento ${ }^{2}$. Tal perspectiva visa desconstruir e substituir os estigmas concernentes aos usuários de substâncias psicoativas como "doentes" e "marginais", concebendo-os como cidadãos de direitos.

Dentre os diversos avanços que a nova legislação proporcionou, destaca-se a assistência universal e integral em saúde para aqueles que sofrem com as necessidades decorrentes do consumo de álcool e outras drogas. Ademais, estruturou-se uma rede de serviços que preza pelo convívio social dos usuários, valoriza a descentralização da atenção e procura ajustar suas ações às reais necessidades da população de modo democrático ${ }^{2}$. Nesse sentido, a implantação de serviços de saúde mental tem crescido significativamente em todo o país desde a aprovação da Lei ${ }^{0}$ 10.216/2011. Registrou-se no ano de 2015 o quantitativo de 1.135 Centros de Atenção Psicossocial (CAPS) do tipo I, 488 do tipo II, 92 do tipo 24h e 210 CAPS tipo infanto-juvenil. Especificamente, existiam 315 do tipo Álcool e Drogas e 88 do tipo Álcool e Drogas 24horas ${ }^{3}$, totalizando 2.328 serviços, 119 a mais que o último quantitativo divulgado pelo relatório Saúde Mental em Dados ${ }^{4}$.

O CAPS Ad é um serviço especializado, de base comunitária, fundamentado nos princípios da Redução de Danos. Em princípio, é um serviço aberto que não impõe nenhuma barreira de acesso, recebendo casos sem agendamento ou usuários sob o efeito de substâncias psicoativas. Sua atuação deve ser interdisciplinar de modo a favorecer o protagonismo dos usuários e priorizar ações em grupo. Suas atividades incluem: consultas individuais, psicoterapia em grupo, medicação assistida ou dispensada, oficinas terapêuticas, visitas domiciliares, atendimento as famílias, reabilitação psicossocial e fornecer alimentação diária ${ }^{5}$. Além dos aspectos relacionados ao campo da atenção, o CAPS Ad também é responsável por organizar a rede local de dispositivos que atendam usuários de substâncias psicoativas no território onde atua, uma vez que coordena atividades de supervisão e matriciamento em saúde mental de outros serviços e programas, bem como equipes de atenção básica $^{2}$.

Para tentar fortalecer as ações em saúde mental foi editada a Portaria $n^{\circ} 3.088 / 2011$, que instituiu a Rede de Atenção Psicossocial (RAPS) e estabeleceu os critérios de organização e implementação em todo o país, integrando a saúde mental em todos os níveis e pontos de atenção no Sistema Único de Saúde (SUS). A RAPS reafirma os princípios da autonomia, o respeito aos direitos humanos e o exercício da cidadania das pessoas com sofrimento mental e com necessidades decorrentes do uso de crack, álcool e outras drogas, há muito defendido pelo Movimento de Luta Antimanicomial e pela Reforma Psiquiátrica. Para tanto, busca promover a equidade e reconhecer os determinantes sociais dos processos saúde-doença-sofrimento-cuidado, além de garantir o acesso 
aos cuidados integrais com qualidade, com ênfase em serviços de base territorial e comunitária, com o estabelecimento de ações intersetoriais em uma rede articulada ${ }^{6}$.

A portaria da RAPS está em consonância com mais dois outros instrumentos com vista a fortalecer a institucionalidade do SUS, que são a Portaria no 4.279/2010 e o Decreto $n^{\circ}$ 7.508/2011. Esses instrumentos abrem uma nova perspectiva para o processo de regionalização da saúde com as Redes de Atenção à Saúde (RAS) e as Regiões de Saúde. A Regionalização da Política de Saúde no Brasil é uma estratégia para superar a fragmentação da oferta de serviços de saúde, por meio da ação cooperativa entre municípios de forma integrada e continuada, aumentando o acesso e a eficiência do sistema?

A Rede de Atenção à Saúde (RAS), instituída pela Portaria nº 4.279/2010, foi definida como conjunto de arranjos organizativos de ações e serviços de saúde, estruturada por diferentes densidades tecnológicas e níveis de complexidade, que integradas por meio de sistemas de apoio técnico, logístico e de gestão, buscam garantir a integralidade do cuidado ${ }^{8}$. Com o propósito de superar a fragmentação histórica da atenção e da gestão do SUS, este desenho permite ofertar uma atenção contínua e integral a determinada população, coordenada pela atenção primária, componentechave para a organização da RAS e reorientação do modelo tecno-assistencial em saúde ${ }^{8,9}$. Para operacionalizar a RAS em todo o país partiu-se da definição das Regiões de Saúde, estabelecidas pelo Decreto $\mathrm{n}^{\mathrm{o}} 7.508 / 2011$, a partir de recortes territoriais constituídos por agrupamentos de municípios limítrofes, com características culturais, econômicas e sociais semelhantes, e composto por redes de comunicação e infraestrutura de transportes compartilhados. Cada Região de Saúde tem a finalidade de integrar a organização, o planejamento e a execução de ações e serviços de saúde, envolvendo diferentes níveis e pontos de atenção (atenção primária; urgência e emergência; atenção psicossocial; atenção ambulatorial especializada e hospitalar e vigilância em saúde) $)^{6}$.

Na prática, o ordenamento da RAPS em cada Região de Saúde passaria pela oferta do cuidado psicossocial em pelo menos sete pontos da rede SUS: 1) Atenção Primária; 2) Atenção Psicossocial Especializada; 3) Atenção à urgência e emergência; 4) Atenção residencial de caráter transitório; 5) Atenção hospitalar; 6) Estratégia de desinstitucionalização; 7) Reabilitação Psicossocial. Tal feito ampliou, sobremaneira, os pontos de acesso e suporte psicossocial à população que passou a contar maior diversidade de serviços implantados nas regiões do país.

Há uma tendência à interiorização da assistência para municípios de médio e pequeno portes, acompanhado da crescente participação da atenção básica em saúde e descentralização dos leitos de atenção psicossocial e Serviços Residenciais Terapêuticos. Isso vem sendo possível em função das mudanças na política de financiamento ao priorizar ações extra hospitalares e de base territorial e comunitária, além das pactuações nas três esferas de governo e níveis de governança regional, por meio das Comissões Intergestores Regionais/CIR e elaboração dos Planos de Ação Regional (PAR) da RAPS em cada Região de Saúde. Vale ressaltar, então, que a regionalização 
da política de saúde no Brasil tem sido uma estratégia fundamental para garantir a organização e oferta dos serviços, acesso, eficiência, redução de custos, aumentar satisfação do usuário, além de diminuir desigualdades, de modo a produzir impactos positivos nas condições sanitárias e na vida das populações ${ }^{3}$. Entretanto, tal política pública vem sendo seriamente ameaçada com as recentes mudanças políticas no país.

Diante isso, objetivamos discutir, a partir da regionalização no SUS, como tem se dado o processo na RAPS, especificamente no âmbito da atenção às pessoas com necessidades decorrentes ao uso de álcool e outras drogas. Esse estudo justifica-se pela possibilidade de contribuir com a literatura que trata sobre a Reforma Psiquiátrica no Brasil e o debate da Regionalização da Saúde Mental após a criação da RAPS. Nesse sentido, objetivamos analisar o processo de regionalização em curso em um dos campos mais tensos do processo reformista que é o da atenção às pessoas com necessidades decorrentes do uso de álcool, crack e outras drogas. E, de forma mais específica, pretendemos dimensionar a RAPS voltada para atenção em álcool e outras drogas, além de problematizar os desafios postos nesse campo com o processo de expansão e de interiorização da atenção psicossocial no Brasil.

\section{DESENVOLVIMENTO}

Trata-se de um estudo transversal, delineado a partir da elaboração de um banco de dados com base nas informações disponibilizadas pela Coordenação Nacional de Saúde Mental e pela plataforma "Observatório Crack é possível vencer". Por meio dessas fontes acessamos as informações dos serviços que integram os principais pontos de atenção da RAPS no âmbito do SUS, voltados para atenção psicossocial em álcool e drogas: atenção básica (Agentes Comunitários de Saúde/ACS, equipes da Estratégia Saúde da Família/ESF, equipes do Núcleo de Apoio à Saúde da Família/NASF e equipes do Consultório na Rua), atenção especializada (CAPS Ad e CAPS Ad 24h), atenção residencial de caráter transitório (Unidade de Acolhimento Adulto e Serviços de Comunidade Terapêutica), atenção hospitalar (leitos psicossociais em hospital geral), além da Rede Intersetorial: Centros de Referência da Assistência Social/CRAS, Centros de Referência Especializado de Assistência Social/CREAS, Centro de Referência para População em Situação de Rua/Centro Pop e Centro Regional de Referência em Crack, Álcool e Outras Drogas/CRR.

Em seguida, as informações foram reconstruídas a partir do banco de indicadores da plataforma Regiões e Redes (http://www.resbr.net.br/), de modo a relacionar os 5.570 municípios brasileiros, organizados em 438 regiões de saúde (Comissões Intergestores Regionais - CIR). Assim, foi possível observar o quantitativo e as modalidades de serviços quanto à cobertura nacional, passíveis de serem desagregados em nível municipal, inclusive pelo porte populacional e as respectivas regiões de saúde que cada município integra. A base de dados resultante foi analisada de forma descritiva com base no software Statistical Package for the Social Sciences (SPSS) for Windows, versão 20. As unidades de análise foram, no caso o mapeamento dos serviços 
da RAPS, a estratificação por estado e porte dos municípios, e no que diz respeito ao processo de Regionalização da Atenção ao álcool e outras drogas no Brasil, as próprias regiões de saúde (CIR).

Para dimensionar a rede voltada para atenção psicossocial em álcool e outras drogas no Brasil, em pelo menos quatro pontos de atenção da RAPS no âmbito do SUS, incluindo também a rede intersetorial (CRAS, CREAS, Centro Pop e CRR), apresentamos as seguintes tabelas:

Tabela 1. Quantitativo de serviços que compõem a rede psicossocial voltada para atenção em álcool e outras drogas no Brasil.

\begin{tabular}{|c|c|c|}
\hline Componentes da rede & Pontos de atenção & $\mathbf{N}$ \\
\hline \multirow{4}{*}{ Atenção Primária em Saúde } & ACS & 332.289 \\
\hline & ESF & 48.410 \\
\hline & Equipe NASF (I, II e III) & 5.067 \\
\hline & Equipe CR & 135 \\
\hline \multirow[t]{2}{*}{ Atenção Psicossocial Especializada } & CAPS Ad & 315 \\
\hline & CAPS Ad 24h & 88 \\
\hline \multirow{2}{*}{ Atenção residencial de caráter transitório } & Unidade de Acolhimento Adulto (UAA) & 38 \\
\hline & Comunidade Terapêutica (CT) & 1.863 \\
\hline Atenção Hospitalar & Leitos em Hospital Geral & 997 \\
\hline \multirow{4}{*}{ Serviços Intersetoriais } & CRAS & 8.155 \\
\hline & CREAS & 2.435 \\
\hline & Centro POP & 235 \\
\hline & CRR & 36 \\
\hline
\end{tabular}

FONTE: Coordenação Nacional de Saúde Mental (Jan/2016). Censo do Sistema Único da Assistência Social de (Brasil, 2015). 
Tabela 2. Dimensionamento dos serviços que compõem a RAPS voltada para atenção em álcool e outras drogas por Regiões do Brasil.

\begin{tabular}{ccccccccc}
\hline & NASF & CAPS AD & CAPS AD 24h & UAA & ECR & Leitos & & CT \\
\hline $\mathbf{N}$ & 385 & 9 & 7 & 2 & 8 & 5 & 11 & 136 \\
$\mathbf{N E}$ & 1.996 & 84 & 25 & 10 & 29 & 15 & 59 & 315 \\
$\mathbf{C O}$ & 368 & 20 & 6 & 1 & 11 & 4 & 12 & 159 \\
$\mathbf{S E}$ & 1.454 & 135 & 29 & 16 & 71 & 72 & 107 & 777 \\
$\mathbf{S}$ & 864 & 67 & 21 & 9 & 16 & 97 & 46 & 476 \\
\hline & $\mathbf{5 . 0 6 7}$ & $\mathbf{3 1 5}$ & $\mathbf{8 8}$ & $\mathbf{3 8}$ & $\mathbf{1 3 5}$ & $\mathbf{1 9 3}$ & $\mathbf{2 3 5}$ & $\mathbf{1 . 8 6 3}$ \\
\hline
\end{tabular}

FONTE: Coordenação Nacional de Saúde Mental (Jan/2016). Censo do Sistema Único da Assistência Social de (Brasil, 2015).

Tabela 3. Dimensionamento dos serviços que compõem a RAPS voltada a atenção ao álcool e outras drogas por Localização e porte populacional.

\begin{tabular}{cccccccccc}
\hline & & NASF & $\begin{array}{c}\text { CAPS } \\
\text { AD }\end{array}$ & $\begin{array}{c}\text { CAPS } \\
\text { AD 24h }\end{array}$ & UAA & ECR & Leitos & & \multirow{2}{*}{ CT } \\
\hline \multirow{3}{*}{ CAPITAL } & & 594 & 61 & 31 & 16 & 66 & 9 & 45 & 306 \\
\cline { 2 - 10 } & PQ & 3.430 & 30 & 15 & 2 & 1 & 121 & 1 & 471 \\
& MPQ & 418 & 74 & 11 & 6 & 4 & 34 & 20 & 365 \\
& MP & 396 & 109 & 10 & 9 & 31 & 21 & 11 & 470 \\
& MGR & 101 & 24 & 13 & 1 & 18 & 4 & 36 & 171 \\
& GR & 128 & 17 & 8 & 4 & 15 & 4 & 22 & 80 \\
\hline Total & & $\mathbf{5 0 6 7}$ & $\mathbf{3 1 5}$ & $\mathbf{8 8}$ & $\mathbf{3 8}$ & $\mathbf{1 3 5}$ & $\mathbf{1 9 3}$ & $\mathbf{2 3 5}$ & $\mathbf{1 . 8 6 3}$ \\
\hline
\end{tabular}

FONTE: Coordenação Nacional de Saúde Mental (Jan/2016). Censo do Sistema Único da Assistência Social de (Brasil, 2015).

Os dados apresentados nas tabelas 1, 2 e 3 indicam o quanto se avançou no país até o momento na estruturação de uma rede diversificada de serviços voltados aos cuidados de pessoas com necessidades decorrentes de álcool e outras drogas. Entretanto, é necessário olhar com cuidado a distribuição desses serviços para observar vários pontos de parada e os desafios do processo de regionalização da RAPS em curso em todo o país.

No que concerne à Atenção Hospitalar, que é um ponto crucial da rede na retaguarda de casos agudos e de quadros clínicos decorrentes do uso abusivo de substâncias, de situações de crise de fissura e/ou de abstinência que demandam urgência psiquiátrica, a rede conta apenas com 997 leitos psicossociais distribuídos em 193 municípios, o que indica somente 3,5\% de cobertura dos municípios de todo o país. Estão concentrados principalmente na região Sul (39,4\%) e Sudeste (35,9\%). Muitos são os desafios para a implantação de leitos psicossociais no Brasil, a exemplo do financiamento, além da resistência por parte de gestores e trabalhadores ancorada na cultura manicomial e no estigma em relação ao usuário de substâncias. Por outro lado, registra-se um maior número de leitos nos municípios do interior $(77,83 \%)$, notadamente de pequeno $(35,1 \%)$, médio pequeno (18\%) e médio porte $(14,14 \%)$, que é um ponto de avanço importante e indica que 
em municípios menores têm havido menor resistência por parte dos gestores na pactuação desse tipo de serviço, especialmente pelos valores de custeio e possíveis incentivos para instalação ou manutenção dos leitos.

Quanto à Atenção Residencial de Caráter Transitório, registra-se 38 UAA que oferecem acolhimento transitório. Funcionam como casas onde pessoas com necessidades decorrentes do uso de crack, álcool e outras drogas, em tratamento nos CAPS, têm apoio profissional e podem viver por um determinado período. É necessário maior incentivo e sensibilização dos gestores para implantação desse serviço, considerando a necessidade de acolhida por um período maior de tempo que tais usuários apresentam. Mesmo em pequeno número, são serviços que estão mais presentes na região Sudeste (42,1\%), seguido do Nordeste (26,3\%), notadamente nas capitais. Dos 133 municípios que atendem ao critério de implantação (200 mil hab.), somente 9,7\% contam o serviço, registrando a necessidade de maior investimento nesse tipo de dispositivo.

Quanto às Comunidades Terapêuticas, mesmo adotando caminho inverso à proposta de Redução de Danos e do Movimento de Luta Antimanicomial, já que, de modo geral, são orientadas pelo paradigma da abstinência como "tratamento" único, além de usarem da disciplina e serem orientadas majoritariamente pela vertente da moral religiosa ${ }^{10}$, tais "serviços", contraditoriamente, compõem a RAPS, a partir da Atenção Residencial de Caráter Transitório. A inclusão das Comunidades Terapêuticas na RAPS gerou inúmeras tensões e descontentamentos entre usuários, familiares e trabalhadores dos serviços de saúde mental. Contudo, acabam respondendo aos apelos da sociedade em geral, entidades religiosas, da assistência social e, até mesmo, de alguns profissionais da área da saúde.

Ao todo são mais de 1.863 CT distribuídas em 713 municípios, localizadas, predominantemente, nas regiões Sudeste $(41,7 \%)$ e Sul $(25,6 \%)$, nos municípios de pequeno $(25,3 \%)$ e médio porte $(25,2 \%)$, conforme figura 1 . Apesar do número existente, de acordo com a Área Técnica de Saúde Mental/DAPES/SAS/MS, nenhum dos serviços, até o momento, tinha apresentado proposta compatível com a Portaria $n^{0} 131$, de 26 de janeiro de 2012, que regula a habilitação para o acolhimento de pessoas em sofrimento por uso de álcool e outras drogas. Os repasses têm se dado pela Secretaria Nacional de Políticas sobre Drogas (SENAD) do Ministério da Justiça, via editais. 
Figura 1. Distribuição de Comunidades Terapêuticas, por municípios, no Brasil.

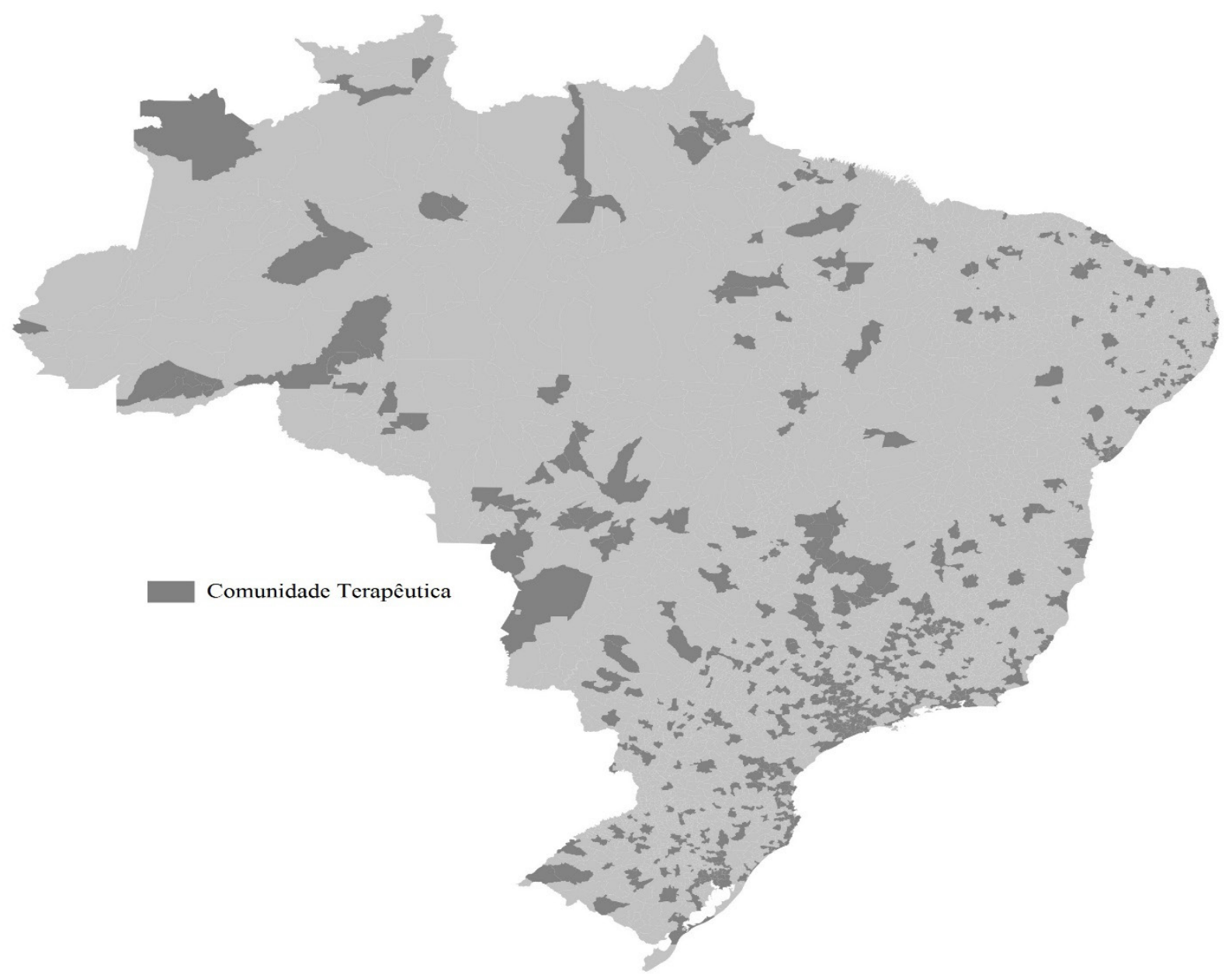

Em relação à Atenção Psicossocial Especializada, por sua especificidade, o CAPS Ad acabou assumindo, historicamente, a função de dispositivo central na rede, local de referência e "porta de entrada" para este tipo de cuidado no SUS. Em função disso, muitos pontos da rede, particularmente a atenção básica e a própria rede intersetorial (assistência social e educação, por exemplo), referenciem casos para serviços do tipo CAPS. Ao dimensionarmos a atenção psicossocial especializada voltada para atenção em álcool e outras drogas, observa-se que é composta por 315 CAPS Ad e 88 CAPS Ad 24h, assim distribuídos no Brasil: 
Figura 2. Distribuição dos CAPS Ad e Ad 24h, por municípios, no Brasil.
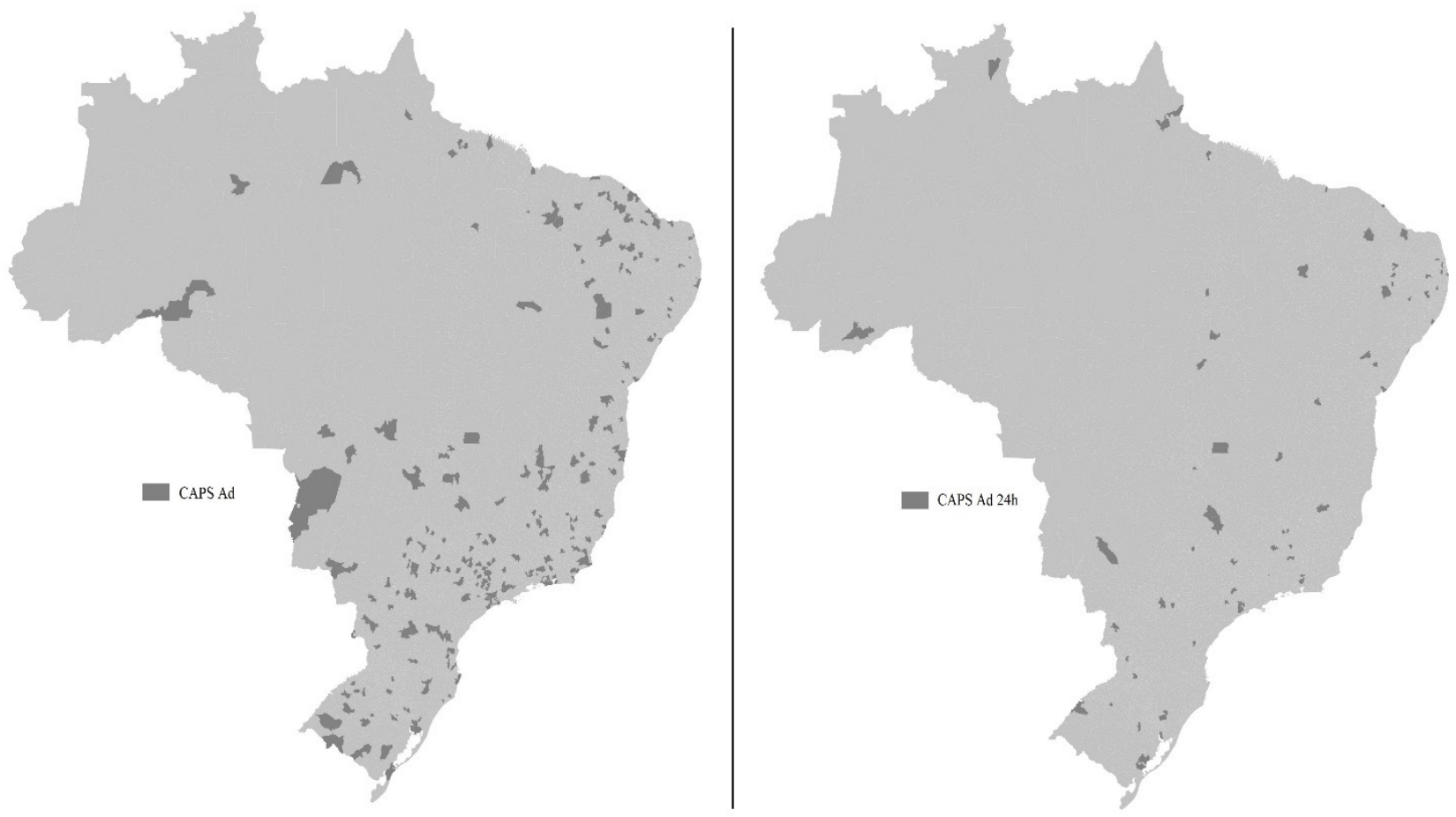

Esses serviços estão em maior númerona região Sudeste, com 42,9\% e 32,9\% respectivamente. A região Norte é a que conta com menor quantidade de CAPS Ad ( $\mathrm{n}=9)$ e a Centro Oeste o de CAPS Ad 24h ( $\mathrm{n}=6)$. O Estado de São Paulo é o que concentra o maior número de serviços em todo país, 24,7\% CAPS Ad e 17,04\% CAPS Ad 24h. Considerando a localização dos serviços, dentre os municípios que atendem ao critério de implantação, 80,63\% dos CAPS Ad e 64,78\% dos CAPS Ad 24h situam-se no interior. Os CAPS Ad se destacam em termos de concentração nos municípios de médio porte $(34,6 \%)$ e médio pequeno porte populacional $(23,49 \%)$, enquanto que os CAPS Ad $24 \mathrm{~h}$ estão presentes em maior quantidade nas capitais $(35,22 \%)$ e nos municípios de pequeno porte (17\%), considerando o caráter de serviço regional nestes casos.

Fica evidente que há um quadro de expansão da rede no país. Alcançou-se o patamar de 268 municípios que contam com CAPS Ad e 68 que contam com CAPS Ad 24h, possibilitando maior oferta de ações de cuidado para pessoas com necessidades decorrentes do uso de substâncias psicoativas no Brasil. Todavia, se considerarmos somente as localidades que possuem os critérios para implantação de CAPS Ad (70 mil hab.) e CAPS Ad 24h (150 mil hab.), há ainda um número significativo de municípios que não contam com esses serviços: registra-se o total de 214 localidades $(50,6 \%)$ que não contam com CAPS Ad e 140 (79,1\%) que não contam com CAPS Ad 24h.

O quadro mais grave pode ser visto em 183 municípios que não contam com ambos os serviços (CAPS Ad e CAPS Ad 24h). Sobre estes últimos, a maioria desses municípios está concentrada nos estados de São Paulo ( $n=48)$, Minas Gerais $(n=25)$, Rio de Janeiro $(n=16)$ e Pará $(n=15)$. Estão localizados principalmente no interior, com destaque para localidades de médio pequeno porte $(n=90)$ e médio porte $(n=86)$. Nesse cenário de desatenção, as Comunidades Terapêuticas acabam 
ocupando um lugar de destaque e cobrindo essa lacuna aberta pela falta de cobertura dos CAPS Ad e Ad 24h em todo território nacional.

Dentre as localidades que contam em sua rede com Comunidades Terapêuticas e outros tipos de CAPS (exceto do tipo álcool e drogas), registra-se o número de 713 municípios, distribuídos nas regiões Sudeste (42,9\%), Sul (25,5\%), Nordeste (18,6\%), Norte $(6,8 \%)$ e Centro-Oeste (6\%). Ademais, chamamos atenção para as localidades que contam com CT e que não há registro, até o momento, de qualquer equipamento tipo CAPS. Trata-se de 195 municípios, distribuídos nas regiões: Sudeste $(n=94)$, Sul $(n=55)$, Nordeste $(n=18)$, Norte $(n=14)$ e Centro-Oeste $(n=14)$, com o destaque de que todos estão localizados no interior, com os seguintes portes: pequeno $(n=175)$, médio pequeno $(n=12)$, médio $(=7)$ e médio grande $(n=1)$. Somente em 16 municípios brasileiros registra-se a presença de CAPS Ad, Ad 24h e CT nos seus territórios, assim distribuídos nas regiões do país: Sudeste $(n=5)$, Nordeste $(n=5)$, Sul $(n=4)$, Norte $(n=1)$ e Centro-Oeste $(n=1)$, sendo nove localizados em capitais e sete no interior. A maior presença das Comunidades Terapêuticas nos municípios menores, em termos populacionais, e a menor presença da rede CAPS nessas localidades, resulta num quadro que acaba por dar maior legitimidade para esse tipo de serviço se consolidar como o principal ponto de acesso à população e resposta ao pânico social que se gerou com chamada "epidemia" do crack por todo o país.

A Atenção Primária, no âmbito da RAPS, abrange quatro componentes, sendo 332.289 Agentes Comunitários de Saúde, 48.410 Equipes Saúde da Família, 5.067 Núcleos de Apoio à Saúde da Família e 135 Equipes de Consultório na Rua. No caso dos três primeiros estão situados em maior número nas cidades de pequeno porte $(46,2 \%, 47,2 \%$ e $67,7 \%)$ e na região Nordeste $(35,3 \%, 36,4 \%$ e 39,4\%). O Consultório na Rua é constituído por equipes móveis de atenção integral à saúde da população em situação de rua, 52,6\% concentram-se na região Sudeste, que possui maior quantitativo de pessoas em situação de rua do país ${ }^{911}$, notadamente nas capitais e municípios de médio porte. Porém, 98,4\% dos municípios não possuem equipe $\mathrm{CR}$, sobretudo, na região Nordeste $(32,4 \%)$.

A Atenção Primária é um dos pontos da rede em que é preciso investir ações de educação permanente e matriciamento em saúde mental, de forma sistemática e continuada, no objetivo de desinstitucionalizar práticas, saberes e, principalmente, a cultura do medo e do preconceito que paira sobre os profissionais em torno das pessoas com necessidades decorrentes do consumo de substâncias psicoativas. Historicamente, os serviços da Atenção Primária foram muito reticentes à oferta de ações em saúde mental no território. O despreparo dos profissionais, da família e da comunidade para lidar com o sofrimento psíquico e com os efeitos decorrentes do uso abusivo e dependente de substâncias têm se tornado cada vez mais evidente ${ }^{12}$.

Estudos constataram despreparo dos profissionais de saúde para acolher e cuidar de tais demandas, sendo que as dificuldades que se interpõem ao acesso e cuidado das manifestações 
de sofrimento que aparecem na APS têm origem diversificada: pouca compreensão a respeito de códigos culturais diferentes para expressar o sofrimento; ausência de capacitações adequadas para as equipes; serviços e profissionais ainda aprisionados no modelo individual e fragmentado de atendimento; prioridade das políticas de saúde mental para as clientelas consideradas graves; ausência de tecnologias de cuidado e de acesso para a clientela com queixas difusas ${ }^{13}$. Observase, atualmente, que a maior parte das propostas de tratamento direcionadas ao consumo abusivo de álcool e outras drogas propõe a institucionalização das pessoas, composta por intervenções médico-farmacológicas, psicossociais, socioculturais ou religiosas. $\mathrm{O}$ usuário tem ficado entre o manicômio/comunidades terapêuticas e o presídio, ocupando o lugar do louco, enquanto sujeito degenerado e/ou destituído de moral-transgressor da lei, ou seja, excluído pela sociedade e rotulado como doente e/ou como delinquente/criminoso ${ }^{14}$.

Nesse sentido, são inúmeros os desafios impostos aos profissionais das equipes de saúde da família (ESF) na atenção em álcool e outras drogas: trabalhar em uma perspectiva diferente daquela aprendida na formação acadêmica (prescritiva e centrada na doença); enfrentar a própria ansiedade, insegurança, preconceito e até a incapacidade para lidar com o usuário de álcool e drogas; apoiar com ações de matriciamento em saúde mental as equipes especializadas; programar atividades com base em políticas ministeriais, que sequer estão consolidadas na maior parte das regiões do país e nem valorizadas pelos gestores locais; criar protocolos de atendimento que permitam o monitoramento e avaliação de ações desenvolvidas junto ao usuário de álcool e outras drogas na região; e, por fim, trabalhar em equipe e em rede de forma a assegurar a integralidade da assistência ${ }^{15}$. É comum também os profissionais da ESF serem procurados para resolver outras questões que podem estar relacionadas ao uso problemático de álcool e outras drogas, logo, é imprescindível o profissional estar preparado e apto para traçar ações de prevenção, promoção, tratamento e reabilitação desses pacientes ${ }^{16}$.

Tais desafios se agravam nas localidades que em se tratando da atenção psicossocial em álcool e outras drogas, contam somente com equipes da Atenção Primária (ACS, ESF e por algum tipo de equipe NASF), como é o caso de pelo menos 3.006 municípios, ou seja, 54\% dos municípios brasileiros. Tais localidades estão assim distribuídas: $31 \%$ na região Sudeste, $28,47 \%$ no Nordeste, $25,15 \%$ no Sul, $8,15 \%$ no Centro Oeste e 7,18\% no Norte. Com relação às ESF, 31 municípios não possuem estas equipes e em 13 municípios a assistência no âmbito da RAPS é feita apenas por Agentes Comunitários de Saúde, o que compromete e fragiliza profundamente o acesso e o acolhimento das demandas dos usuários e familiares no campo da atenção psicossocial em álcool e outras drogas.

Especificamente sobre as equipes NASF, que foram criadas como estratégia de ampliação da diversidade e da abrangência das ações realizadas pela ESF, é preciso situar alguns aspectos positivos e outros que consideramos como pontos de obstáculo e parada importantes no processo expansão e interiorização da RAPS no país. Tais equipes têm como meta desenvolver ações de 
matriciamento, apoiar as equipes de atenção primária na discussão dos casos, no atendimento compartilhado e na construção conjunta de projetos terapêuticos singulares, visando à coordenação e continuidade do cuidado.

De acordo com o Ministério da Saúde há três modalidades de NASF: a) tipo 1 - composto de no mínimo cinco profissionais e devendo assistir a pelo menos oito equipes da ESF; b) tipo 2 voltado para municípios com menos de dez habitantes por quilômetro quadrado, devendo incluir no mínimo três profissionais de nível superior de ocupações não-coincidentes e tendo que desenvolver ações com pelo menos três equipes da ESF; c) tipo 3 - criado pela Portaria $n^{0}$ 3.124/2012 para atenção integral a usuários de crack, álcool e outras drogas, em municípios com menos de 20.000 habitantes ${ }^{17}$.

Das 5.067 equipes NASF implantadas em todo o país até o momento, 2.962 são do tipo NASF1, 976 do tipo NASF2 e 1.129 do tipo NASF3. Diferentemente das equipes NASF1, 20\% localizadas nas capitais e $80 \%$ nos municípios do interior, com destaque para as cidades de pequeno porte $(45,23 \%)$, as equipes NASF3 estão todas nos municípios do interior e $99,8 \%$ nas cidades de pequeno porte. Este último dado é um excelente indicador considerando que $54 \%$ dos municípios brasileiros no âmbito da atenção a usuários de álcool e outras drogas contam somente com a Atenção Primária.

Figura 3. Distribuição dos tipos de NASF, por municípios, no Brasil.

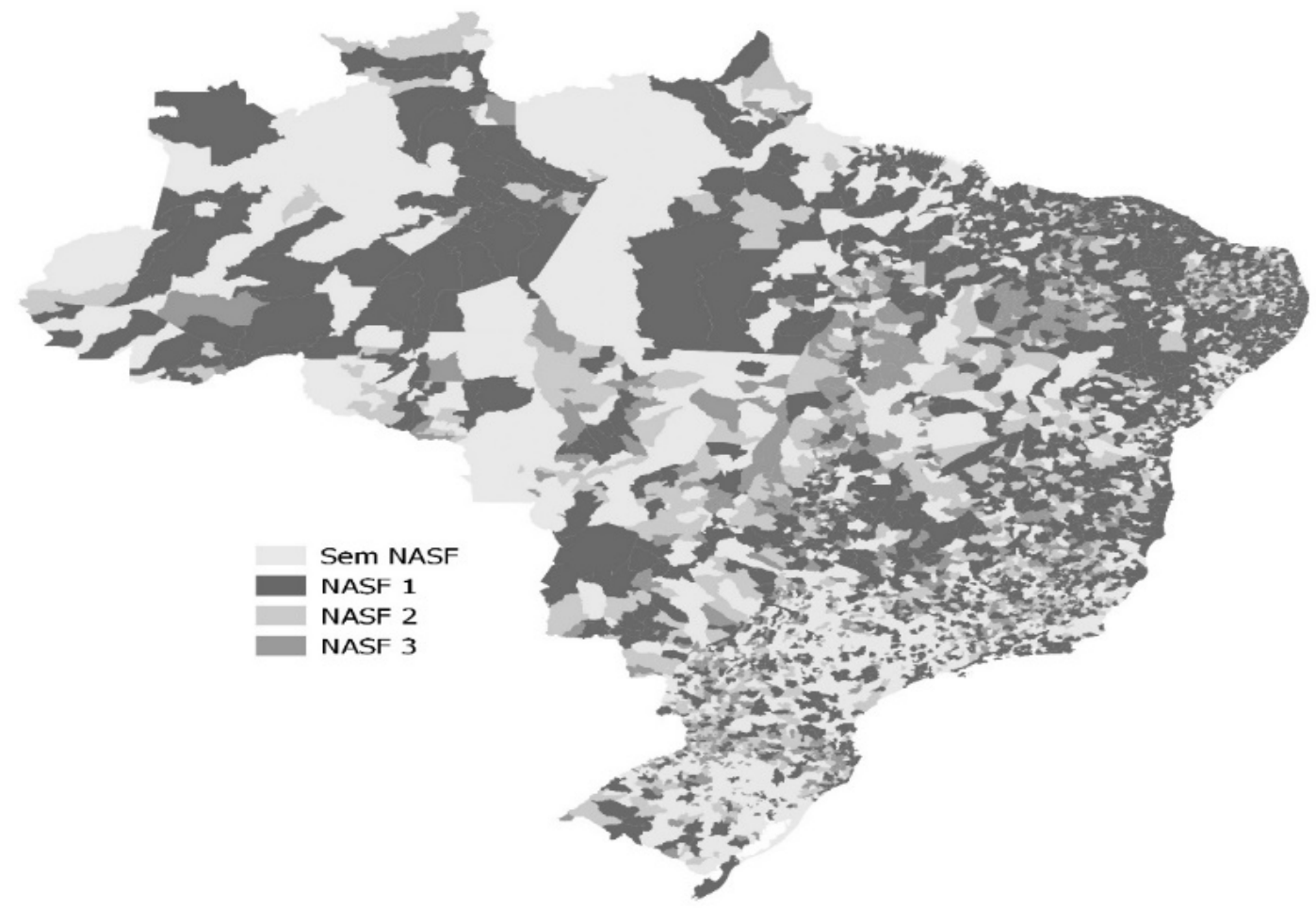

Há 1.798 localidades que não possuem qualquer tipo de NASF, condição que fragiliza ISSN 1982-8829 Tempus, actas de saúde colet, Brasília, 11(3), 144-162, 2017 - Epub mar, 2018 
fortemente a RAPS nos municípios pequenos. Apesar dos avanços na cobertura desses serviços, do convívio com pessoas usuárias de álcool e outras drogas, é notório o pouco envolvimento dos profissionais, tanto na ESF, quanto no NASF na questão18. Há preconceito, inexperiência, pouca proximidade com a área de atuação, encontram-se marcados pelo modelo biomédico, de modo que o envolvimento desses profissionais ainda muito incipiente19. Como consequência disso, a população e os próprios serviços que compõem a atenção primária nessas localidades, acabam recorrendo às Comunidades Terapêuticas da região como único recurso de cuidados.

Além da rede SUS, o campo de proteção, atenção e cuidado voltado para as pessoas com necessidades decorrentes do uso de substâncias psicoativas é composto de serviços da rede intersetorial envolvendo a educação, assistência social, justiça e direitos. Para efeito deste estudo, circunscrevemos o levantamento em torno dos equipamentos da Assistência Social e dos Centro Regional de Referência em Crack, Álcool e Outras Drogas/CRR.

No âmbito do Sistema Único da Assistência Social, há 8.155 CRAS, 2.435 CREAS, 36 CRR e 235 Centros POP. O CRAS é um serviço caracterizado como porta de entrada da Assistência Social. Encontra-se em áreas de maior vulnerabilidade social, com o objetivo de fortalecer a convivência com a família e com a comunidade. Busca assegurar o acesso da população aos serviços, benefícios e projetos de assistência social; atua junto à comunidade para o enfrentamento de problemas sociais, como falta de acessibilidade, de transporte, espaços de lazer e de cultura, baixa qualidade na oferta de serviços, violência no bairro, trabalho infantil, dentre outros. Esse serviço está distribuído notadamente nas regiões Sudeste $(34,4 \%)$ e Nordeste $(32,1 \%)$, nas capitais $(1,75 \%)$, e municípios de pequeno $(49,1 \%)$, médio pequeno $(17,5 \%)$ e grande porte $(18,7 \%)$.

O CREAS oferta serviço especializado de maneira contínua à famílias e indivíduos (crianças, adolescentes, jovens, adultos, idosos, mulheres) que se encontram em situação de ameaça ou violação de direitos, tais como violência física, psicológica, sexual, tráfico de pessoas, cumprimento de medidas socioeducativas em meio aberto, situação de risco pessoal e social associados ao uso de drogas, etc. A região Nordeste $(38,1 \%)$, seguida da Sudeste $(28,4 \%)$ destacam-se pelo maior número de serviços, notadamente, os municípios de pequeno e médio pequeno porte. Mas, vale pontuar que $60,6 \%$ dos municípios brasileiros não são assistidos por esse serviço, na sua totalidade municípios do interior e de pequeno porte.

O Centro POP é um espaço de referência e Serviço Especializado para pessoas em situação de rua que promove o convívio grupal, social e o desenvolvimento de relações de solidariedade, afetividade e respeito. Concentra-se na região Sudeste (45,5\%), principalmente, nas capitais e municípios de médio grande porte. Somente 60 municípios contam com os dois serviços voltados para população em situação de rua em sua rede (ECR e Centro Pop), assim distribuídos: Sudeste $(n=25)$, Nordeste $(n=14)$, Sul $(n=10)$, Norte $(n=6)$ e Centro Oeste $(n=5)$. A relação capital interior é de 21 para 39.

ISSN 1982-8829 Tempus, actas de saúde colet, Brasília, 11(3), 144-162, 2017 - Epub mar, 2018 
O CRR destina-se à formação presencial permanente dos profissionais que atuam nas redes de saúde, assistência social e segurança pública, bem como daqueles que atuam no Ministério Público, Poder Judiciário e entidades que prestam apoio a adolescentes em cumprimento de medidas socioeducativas e estão em instituições. Atua em locais com uma população igual ou superior a 500 (quinhentos) mil habitantes ou em municípios menores quando firmadas parcerias com municípios vizinhos para atender população de tamanho semelhante. Logo, esse serviço encontra-se com destaque nas regiões Sudeste (44,4\%) e Nordeste (27,7\%), nas capitais, municípios de médio e médio grande porte.

Em síntese, não há dúvida quanto ao avanço em termos da estruturação de serviços psicossociais voltados para os cuidados em álcool e outras drogas no Brasil, especialmente após implantação da RAPS. Ações de caráter intersetorial e regionalizado foram fortalecidas, apesar das lacunas e dos desafios recorrentes que esse campo impõe à abertura de serviços especializados em localidades com desequilíbrio no desenvolvimento regional e baixa concentração territorial, que contam, na maior parte dos casos, apenas com a retaguarda da atenção primária. É evidente a necessidade de aprofundamento de ações de educação permanente, com vistas a fortalecer o trabalho em rede entre os serviços e demais atores/organizações presentes no território cotidiano dos usuários. Sendo um país de proporções continentais, essas fragilidades em termos de cobertura e oferta de serviços na rede especializada, produz um profundo desamparo entre usuários, familiares e trabalhadores da saúde mental. Isso se agrava com os problemas da atenção básica e com a fragmentação histórica das redes de saúde e intersetoriais no país.

Em função disso, o debate sobre a regionalização da saúde no Brasil, proposto desde a emergência do SUS, ganhou mais força a partir dos anos 2000, cuja maior inovação foi o ano de 2006, proporcionado pelo Pacto da Saúde. A regionalização é entendida como proposta para superar a fragmentação sistêmica da oferta de serviços de saúde, por meio da ação cooperativa entre municípios de forma integrada e continuada, aumentando o acesso e a eficiência do sistema $^{20}$, fortalecendo respostas regionais a problemas locais ${ }^{21}$ e reduzindo as iniquidades inter e intrarregionais de modo a ampliar o acesso integral à população.

Muito se avançou na regionalização da saúde, com a institucionalização de espaços interfederativos para fomentar a regionalização de maneira cooperativa, mecanismos de negociação e pactuação dos compromissos regionais e ferramentas para avaliação das regiões de saúde, especialmente com a introdução do Contrato Organizativo da Ação Pública - COAP, instrumento de pactuação entre gestores para constituir as redes assistenciais para integrar o cuidado e atender as necessidades em saúde da população no seu território ${ }^{22}$. Assim, divide-se a política de regionalização em dois momentos: a) o Pacto e o estabelecimento do COAP, com intensas discussões em torno do tema da regionalização e as primeiras respostas no âmbito gerencial e assistencial no âmbito das redes descentralizadas de atenção à saúde; b) e o momento posterior, de esvaziamento institucional 
e ausência de inovação na temática da regionalização, em função da estagnação econômica, da forte desestruturação fiscal dos entes governamentais e ruptura democrática com o impedimento da presidente da República Dilma Rousseff eleita em 2014.

Atualmente, estamos em um dos períodos mais delicados da nossa história depois da retomada da democracia em 1988, em que sob o mandato ilegítimo do vice-presidente Michel Temer desde 2016, estabeleceu-se Novo Regime Fiscal (NRF) para o país, acompanhado de uma série de contrarreformas que atingem diretamente o setor Saúde, com ações sistemáticas de desmonte do SUS, a exemplo da alteração da Política Nacional da Atenção Básica, com indicativo claro de alteração na Política de Saúde Mental. Ou seja, estamos observando a mudança de rumos com o plano de governo alinhado a uma pauta de austeridade fiscal, barganha política e conservadora.

Nesse confuso cenário é possível detectar avanços em relação à regionalização da saúde. Porém, não podemos dizer o mesmo quanto a regionalização da RAPS no Brasil. Há pontos de parada que são muito problemáticos para atenção psicossocial ${ }^{3}$. Atualmente são 438 regiões de saúde que agrupam os 5.570 municípios existentes no país. Apesar de 95,43\% $(n=418)$ das regiões de saúde serem cobertas pela RAPS, se considerarmos pelo menos dois pontos de atenção (APS com equipes ACS, ESF e NASF e rede CAPS independente da modalidade), áreas completamente desassistidas. Assim:

Nas regiões com critério de implantação para CAPS I (entre 15 e 70 mil habitantes), identificamos 19 CIR que deveriam ser constituídas por esses dois pontos de atenção (APS e CAPS I), porém, em pelo menos quatro regiões $(21,05 \%)$ não há registro de qualquer serviço tipo CAPS para retaguarda, somente serviços da atenção primária. Nas regiões com critério de implantação para CAPS II (entre 70 e 150 mil habitantes), identificamos 77 CIR que deveriam contar com os mesmos dois pontos de atenção (APS e CAPS II), sendo que em pelo menos 58 regiões $(75,32 \%)$ não há registro deste serviço em específico, apesar de contarem com outras modalidades de CAPS, especialmente do tipo I. Naquelas com critério de implantação para CAPS III (acima de 150 mil habitantes), identificamos 342 CIR que deveriam ser constituídas pelo menos por dois pontos de atenção (APS e CAPS III), porém, 296 regiões (86,55\%) não contam com este serviço em específico, apesar de registrarem outras modalidades de CAPS nos territórios ${ }^{3}$.

Além disso, 13 regiões de saúde contam somente com equipes de ACS, ESF ou NASF como único ponto de acesso à saúde mental em seus territórios ${ }^{3}$, sendo um ponto gravíssimo de desassistência e iniquidade no acesso à rede psicossocial, pois os usuários nem contam com a retaguarda de serviços especializados no seu município, muito menos nos municípios próximos que compõem a mesma região de saúde, tendo que recorrer a municípios mais distantes para obter algum tipo de assistência mais especializada.

No caso dos CAPS Ad e Ad 24h, são 200 regiões de saúde do país (45,16\%) que não contam com acesso a esses serviços, mesmo com a implementação das ações do programa Crack, é Possível Vencer. É um quadro extremamente preocupante e revela a fragilidade da atenção regionalizada às pessoas com necessidades decorrentes do uso de álcool e outras drogas. Essa situação é o que vem 
estimulando o aumento das comunidades terapêuticas, as quais não sofrem regulação nenhuma do estado e nem têm compromisso com as diretrizes da atenção psicossocial e da reforma psiquiátrica.

No relatório de gestão realizado da Coordenação Nacional de Saúde Mental do Ministério da Saúde referente ao período de 2011-2015, somente 169 Planos de Ação Regional (PAR) da RAPS, ou cerca $35 \%$, tinham sido pactuados em todo país. Os níveis de governança, de cofinanciamento e de legitimidade dos referidos planos regionais como instrumento de gestão interfederativo em cada CIR apresentam-se como os grandes desafios para avançar na efetivação da RAPS de forma regionalizada em todo país ${ }^{23}$. Esse dado reforça ainda mais o entendimento de que vivemos uma situação de risco de recrudescimento dos pontos de parada e obstáculos na política de regionalização, ou de reduzi-la ao aspecto meramente racional-instrumental, visando à eficiência administrativa e redução de custos, sendo operacionalizada por ações protocolares, regulatórias, burocráticas e pouco comprometida com a efetivação dos princípios do SUS ${ }^{22}$.

\section{CONCLUSÃO}

Esse trabalho teve como foco a atenção psicossocial voltada para usuários com necessidades decorrente do uso de álcool, crack e outras drogas. Apesar dos esforços de efetivação da Política Nacional de Atenção Integral ao Uso de Álcool e Outras Drogas, em consonância a Lei da Reforma Psiquiátrica no Brasil ( $\mathrm{N}^{\mathrm{o}}$ 10.216/2001), apontamos, por meio de metodologia de cruzamento de informações acerca dos serviços voltados à essa população e com dados das regiões de saúde, lacunas importantes quanto à estruturação de uma rede com caráter regionalizada em todo o país. Destacamos também a necessidade de articulação entre os diversos níveis que compõem a atenção em saúde mental na RAPS, bem como com outros dispositivos do território na perspectiva da intersetorialidade com as redes de educação, assistência social e justiça/campo de direitos.

No âmbito da regionalização da RAPS ainda não consolidamos uma cultura institucional no Brasil, com também no âmbito das políticas de saúde voltadas para os processos de planejamento regional como instrumento de definição e ordenamento do SUS ${ }^{24}$ Como resultado, o que se vê, é uma rede de serviços insuficiente e pouco organizada. Há uma “expansão errante', que não considera o planejamento e a pactuação regional necessários para a sustentabilidade do sistema" 25 . Assim, é preciso superar a burocracia e o enrijecimento das CIR e dos grupos condutores da RAPS, com Planos de Ação Regionais que visem ao planejamento dos sistemas regionais a médio e longo prazos.

Por fim, chamamos atenção que o debate sobre a regionalização da RAPS precisa ser inserida na agenda de discussão dos trabalhadores e gestores da Saúde Mental para fortalecer em cada região de saúde as ações voltadas à mudança do modelo tecnoassistencial em saúde, em especial no âmbito da atenção em álcool e drogas.

ISSN 1982-8829 Tempus, actas de saúde colet, Brasília, 11(3), 144-162, 2017 - Epub mar, 2018 


\section{REFERÊNCIAS}

1. Teixeira MB, de Leão Ramôa M, Engstrom E, Ribeiro JM. Tensões paradigmáticas nas políticas públicas sobre drogas: análise da legislação brasileira no período de 2000 a 2016. Ciê Saú Col. 2017; 22(5): 1455-1466.

2. Brasil, Ministério da Saúde (MS). A política do Ministério da Saúde para a atenção integral a usuários de álcool e outras drogas. Brasília: Ministério da Saúde; 2003.

3. Macedo JP, de Abreu MM, Fontenele MG, Dimenstein M. A regionalização da saúde mental e os novos desafios da Reforma Psiquiátrica brasileira. Saú Soc. 2017; 26(1): 155-170.

4. Brasil, Ministério da Saúde (MS). SAS/Dapes. Coordenação Geral de Saúde Mental, Álcool e Outras Drogas. Saúde Mental em Dados. 2015; 10(12). [Acesso em 2017 junho 22]. Disponível em: https://goo.gl/ULv73a.

5 Brasil. Ministério da Saúde. Portaria n ${ }^{0}$ 3.088, de 23 de dezembro de 2011. Institui a Rede de Atenção Psicossocial para pessoas com sofrimento ou transtorno mental e com necessidades decorrentes do uso de crack, álcool e outras drogas, no âmbito do Sistema Único de Saúde (SUS) e dá outras providências. Diário Oficial da União, Brasília, DF; 2011.

6. Brasil, Ministério da Saúde (MS). Decreto no 7.508, de 28 de junho de 2011. Regulamenta a Lei $\mathrm{n}^{\circ}$ 8.080, de 19 de setembro de 1990, para dispor sobre a organização do SUS, o planejamento da saúde, a assistência à saúde ea articulação interfederativa, e dá outras providências. Diário Oficial da União; 2011.

7. Santos L, de Sousa Campos GW. SUS Brasil: a região de saúde como caminho. Saú Soc. 2015; 24(2): 438-446.

8. Brasil, Ministério da Saúde (MS). Portaria no 4.279, de 30 de dezembro de 2010. Estabelece diretrizes para a organização da Rede de Atenção à Saúde no âmbito do Sistema Único de Saúde (SUS). Diário Oficial da União; 2010.

9. Mendes EV. As redes de atenção à saúde. Ciênc. saúde coletiva. 2010; 15(5): 2297-2305. [Acesso em 2017 junho 22]. Disponível em: http://www.scielo.br/scielo.php?script=sci_arttext\&pid=S1413-81232010000500005\&lng=en.

10. Méllo RP. As drogas cotidianas em tempos de sobrevivência. In: Vieira LLF, Rios LF, Queiroz TN (Orgs). A problemática das drogas: contextos e dispositivos de enfrentamento. Recife: Editora UFPE; 2016: 20-52.

11. Brasil, Ministério do Desenvolvimento Social e de Combate à Fome. Pesquisa nacional sobre a população em situação de rua. Brasília; 2008 .[Acesso em 2017 março 22]. Disponível em: https://goo.gl/kN8dAq.

12. Silveira MR. A saúde mental na atenção básica: um diálogo necessário (Tese de doutorado). Universidade Federal de Minas Gerais, Belo Horizonte; 2009.

13. Tavares ALB, de Souza AR, Pontes RJS. Estudo da demanda de saúde mental em Centro de Saúde da Família em Caucaia, Ceará, Brasil. Rev Bra Med Fam Comun. 2013; 8(26): 35-42.

14. Vasconcelos Rosenstock KI, Neves MJD. Papel do enfermeiro da atenção básica de saúde na abordagem ao dependente de drogas em João Pessoa, PB, Brasil. Rev Bra Enf. 2010; 63(4).

15. Gonçalves AM. Cuidados diante do abuso e da dependência de drogas: desafio da prática do Programa Saúde da Família (Doctoral dissertation, Universidade de São Paulo); 2002.

ISSN 1982-8829 Tempus, actas de saúde colet, Brasília, 11(3), 144-162, 2017 - Epub mar, 2018 
16. Almeida DAD. O apoio matricial sobre álcool e outras drogas integrando a saúde mental na atenção primária à saúde; 2016.

17. Brasil. Ministério da Saúde. Portaria n 3.124,de 28 de dezembro de 2012. Redefine os parâmetros de vinculação dos Núcleos de Apoio à Saúde da Família (NASF) Modalidades 1 e 2 às Equipes Saúde da Família e/ou Equipes de Atenção Básica para populações específicas, cria a Modalidade NASF 3, e dá outras providências. Brasília: Ministério da Saúde.

18. Lima AIO, Dimenstein M, Macedo JP. Consumo de álcool e drogas e o trabalho do psicólogo no núcleo de apoio à saúde da família. Psicologia em Pesquisa, UFJF. 2015; 9(2): 188-197.

19. Rodrigues CNDA. A redução de danos na problemática do álcool, crack e outras drogas sob a ótica dos profissionais do núcleo de apoio à saúde da família no município de Fortaleza (Doctoral dissertation); 2016.

20. Santos L, de Sousa Campos GW. SUS Brasil: a região de saúde como caminho. Saúde e Sociedade. 2015; 24(2): 438-446.

21. Ribeiro PT. Perspectiva territorial, regionalização e redes: uma abordagem à política de saúde da República Federativa do Brasil. Saúde e Sociedade. 2015; 24(2): 403-412.

22. Viana ALA, Ferreira MP, Cutrim MAB, Fusaro ER, Souza MR, Mourão L, Chancharulo AP, Mota PHS. Política de regionalização do SUS em debate: avanços e impasses da implementação das regiões e redes no Brasil. Novos Caminhos, n.15. Pesquisa Política, Planejamento e Gestão das Regiões e Redes de Atenção à Saúde no Brasil. [Acesso em 2017 junho 23]. Disponível em: www.regiaoeredes.com.br

23.Brasil. Ministério da Saúde. Secretaria de Atenção à Saúde. DAPES. Coordenação Geral de Saúde Mental, Álcool e Outras Drogas. Saúde Mental no SUS: Cuidado em Liberdade, Defesa de Direitos e Rede de Atenção Psicossocial. Relatório de Gestão 2011/2015. Ministério da Saúde: Brasília. 2016: 143.

24. Mello GA, Pereira APCM, Uchimura LYT, Iozzi FL, Demarzo MMP, Viana ALD. O processo de regionalização do SUS: revisão sistemática. Ciên \& Saú Col. 2017; 22(4): 1291-1310.

25. Reis AAC, Sóter APM, Furtado LAC, Pereira SSS. Reflexões para a construção de uma regionalização viva. Ciên \& Saú Col. 2017; 22(4): 1045-1054.

Artigo apresentado em 30/10/2017

Artigo aprovado em 08/01/2018

Artigo publicado no sistema em 16/04/2018

ISSN 1982-8829 Tempus, actas de saúde colet, Brasília, 11(3), 144-162, 2017 - Epub mar, 2018 